\title{
Entransy dissipation and irreversibility of some thermodynamic processes
}

\author{
WANG WenHua, CHENG XueTao* \& LIANG XinGang \\ Key Laboratory for Thermal Science and Power Engineering of Ministry of Education, Department of Engineering Mechanics, Tsinghua \\ University, Beijing 100084, China
}

Received May 12, 2012; accepted July 26, 2012

\begin{abstract}
The relationship between entransy dissipation and the irreversibility of some thermodynamic processes, such as heat transfer, work-heat conversion, free expansion, isothermal diffusion etc., are analyzed in this paper. The results show that there is entropy generation but no entransy dissipation in irreversible work-heat conversion, free expansion and isothermal diffusion. Therefore, entransy dissipation cannot be used to describe the irreversibility of these processes. Both entropy generation and entransy dissipation exist in heat transfer process, which indicates that the entransy dissipation can be used to describe the irreversibility of heat transfer processes. Furthermore, the irreversibility of endoreversible cycles is analyzed. As all the irreversibility in endoreversible cycles is attributed to heat transfer between the heat sources and the working medium, entransy dissipation can be used to describe the irreversibility of this kind of cycles. To verify this conclusion, numerical examples of the endoreversible Carnot cycle are discussed.
\end{abstract}

entransy dissipation, entropy generation, irreversibility, endoreversible thermodynamic cycles

Citation: Wang W H, Cheng X T, Liang X G. Entransy dissipation and irreversibility of some thermodynamic processes. Chin Sci Bull, 2012, 57: 4091-4099, doi: $10.1007 / \mathrm{s} 11434-012-5450-2$

Irreversibility is one of the common features of thermodynamic processes in nature. For instance, mechanical work can be totally transformed into heat by means of rubbing in heat-work conversion process, but heat cannot be totally turned into mechanical work. In diffusion processes, two kinds of fluid in a mixture cannot separate from each other spontaneously. In heat transfer processes, heat can only be transferred from a high temperature object to a low temperature one but cannot be transferred from a low temperature object to a high temperature one spontaneously. The equivalence of the irreversibility of these thermodynamic processes is proved strictly [1,2]. For these thermodynamic processes, the irreversibility can be described by entropy generation $[1,3,4]$. Researches show that entropy generation is always accompanied by irreversible thermodynamic processes, and larger entropy generation means more irreversibility of the thermodynamic process.

*Corresponding author (email: chengxt06@mails.tsinghua.edu.cn)
In the history of science, some principles have had more than one expression. In thermodynamics, for instance, the Clausius expression and the Kelvin expression both describe the second law of thermodynamics even though the expressions are different. In quantum mechanics, the Schrödinger equation and the matrix mechanics reveal the quantum world in different ways [5]. In recent years, researches show that the irreversibility in heat transfer processes can be described by entransy dissipation besides entropy generation [6-9]. Entransy was proposed by Guo et al. [6] to describe the potential energy of heat transfer by comparing electricity and heat transfer. If a body has an internal energy $U$ and a temperature $T$, its entransy is defined as [6]

$$
G=\frac{1}{2} U T \text {. }
$$

Guo et al. [6] and Han et al. [7] analyzed the thermal equilibrium process of two bodies with different initial temperature in an isolated system. Their results show that entransy 
decreases after thermal equilibrium, indicating that there is entransy dissipation in thermal equilibrium process. Cheng et al. [8] derived the relationship between entransy and microstate number, and analyzed the microstate number of thermal equilibrium process. The results show that the microstate number increases and the entransy decreases in thermal equilibrium process. Furthermore, Cheng et al. [9] analyzed the entransy change when a small amount of heat is exchanged in an isolated system based on the second law of thermodynamics, and proved that the entransy dissipation always exists in heat transfer processes. Therefore, entransy dissipation can describe the irreversibility of heat transfer processes.

The principle of extremum entransy dissipation and the principle of minimum thermal resistance are developed based on the concept of entransy dissipation [6,10,11]. These principles have been applied to the optimizations of heat conduction [6,10-14], heat convection [6,15], thermal radiation [16,17], heat exchangers [18-23] and heat exchanger networks [24].

At present, the concept of entransy dissipation is mainly applied to the analyses of heat transfer irreversibility and the optimization of heat transfer processes. In addition, Chen et al. [25] found that the principle of extremum entransy dissipation is not suitable for optimizing the design of heat exchangers when heat is transferred for doing work. Taking the Carnot cycle as an example, $\mathrm{Xu}$ et al. [26,27] analyzed the entransy and entransy dissipation with heat-work conversion process from the second law of thermodynamics. Chen et al. [28] proposed the extended concept of entransy, mass entransy, and developed the extremum mass entransy dissipation principle, which is fit for the optimization of mass transfer process. Based on the concept of mass entransy, Xia et al. [29] discussed the optimization of constant-temperature mass transfer process. Cheng et al. $[30,31]$ furthermore extended the concept of entransy, and defined some kinds of potential energies as potential entransy. The principles of potential entransy are obtained in generalized flow. However, it is still necessary to discuss whether entransy dissipation can describe the irreversibility of thermodynamic processes such as work-heat conversion and diffusion, etc. This paper will discuss and clarify the relationship between entransy dissipation and some irreversible processes.

\section{Irreversibility analysis of heat transfer, work- heat conversion, free expansion and isothermal diffusion processes}

From the viewpoint of thermodynamics, heat transfer, workheat conversion, free expansion of ideal gas and isothermal diffusion processes are all irreversible. The entropy generation and the entransy dissipation of these typical irreversible processes are analyzed and discussed, and the relationship between entransy dissipation and the irreversibility of thermodynamic processes is obtained.

\subsection{Heat transfer process}

Let us analyze a heat transfer process in an isolated system without output work interaction. As shown in Figure 1, the isolated system is composed of two parts whose volumes are invariant. Their initial temperatures are $T_{1}$ and $T_{2}$ respectively. There is a plate with ideal heat insulation between the two parts. We assume that there is no energy transfer between the parts before the plate is taken away. At the instant when the plate is removed, heat transfer starts between the parts. A small amount of heat $\delta Q$ is transferred from part 1 to part 2 in a certain period of time.

In order to examine the irreversibility of heat transfer processes, the entropy and entransy before and after $\delta Q$ is transferred are analyzed and discussed for three cases: the heat capacities of the two parts are finite, the heat capacity of one part is infinite while that of the other one is finite, and the heat capacities of the two parts are both infinite. For the three cases, entropy generation unavoidably accompanies heat transfer processes according to non-equilibrium thermodynamics [32], which indicates that these physical processes are irreversible.

For the case that both the heat capacities of the two parts are finite, we assume the heat capacity and the mass of part 1 are $c_{1}$ and $m_{1}$ respectively, and those of part 2 are $c_{2}$ and $m_{2}$ respectively. Cheng et al. [9] calculated the entransy change of the system after $\delta Q$ is exchanged:

$$
\Delta G=\frac{1}{2} \delta Q\left[\kappa \delta Q+2\left(T_{2}-T_{1}\right)\right]
$$

where $\kappa=1 / c_{1} m_{1}+1 / c_{2} m_{2}$. They proved that for spontaneous heat transfer process [9]

$$
\Delta G<0 .
$$

It can be found that the entransy decreases during heat transfer process. For the case in which the heat capacities of both subsystems are finite, there is entransy dissipation besides entropy generation in the irreversible heat transfer processes.

For the second case, assume that the heat capacity of one part is infinite whose temperature remains constant during heat transfer process. The heat capacity and mass of part 1

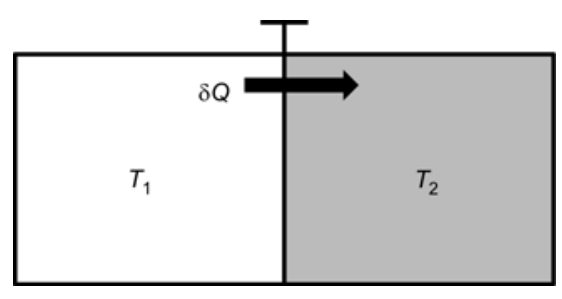

Figure 1 Heat transfer process in an isolated system. 
are $c_{1}$ and $m_{1}$ respectively, and the heat capacity of part 2 is infinite. The temperature of part 1 after $\delta Q$ is transferred is

$$
T_{1}^{\prime}=\left(c_{1} m_{1} T_{1}-\delta Q\right) /\left(c_{1} m_{1}\right) .
$$

The entransy of part 1 before and after heat transfer are

$$
\begin{gathered}
G_{1}=\frac{1}{2} c_{1} m_{1} T_{1}^{2}, \\
G_{1}^{\prime}=\frac{1}{2} c_{1} m_{1} T_{1}^{\prime 2}=\frac{1}{2} c_{1} m_{1}\left[T_{1}-\delta Q /\left(c_{1} m_{1}\right)\right]^{2} .
\end{gathered}
$$

The entransy change of part 1 is

$$
\Delta G_{1}=G_{1}^{\prime}-G_{1}=\frac{1}{2} \frac{\delta Q^{2}}{c_{1} m_{1}}-T_{1} \delta Q .
$$

As the temperature of part 2 remains constant during the heat transfer process, entransy change is

$$
\Delta G_{2}=T_{2} \delta Q .
$$

Therefore, the entransy change of the whole system is

$$
\begin{aligned}
\Delta G & =\Delta G_{1}+\Delta G_{2}=\frac{1}{2} \delta Q\left[\frac{\delta Q}{c_{1} m_{1}}-2\left(T_{1}-T_{2}\right)\right] \\
& =\frac{1}{2} \delta Q\left\{\left[\frac{\delta Q}{c_{1} m_{1}}-\left(T_{1}-T_{2}\right)\right]-\left(T_{1}-T_{2}\right)\right\} .
\end{aligned}
$$

According to the second law of thermodynamics, if $\delta Q>$ 0 , we have

$$
T_{1}>T_{2}, \quad T_{1}^{\prime} \geqslant T_{2} .
$$

Substituting eq. (4) into eq. (10) yields

$$
T_{1}-T_{2}>\delta Q /\left(c_{1} m_{1}\right) \text {. }
$$

By combining eqs. (9)-(11), we could find that eq. (9) is negative. There must be entransy dissipation in this irreversible heat transfer process. We can get the same conclusion if $\delta Q<0$.

For the third case, the heat capacities of the two parts are both infinite and their temperatures remain constant during the heat transfer process. The entransy change of part 2 in heat transfer process can be expressed by eq. (8), while the entransy change of part 1 is

$$
\Delta G_{1}=-T_{1} \delta Q .
$$

The entransy change of the whole isolated system is

$$
\Delta G=\Delta G_{1}+\Delta G_{2}=\left(T_{2}-T_{1}\right) \delta Q .
$$

If $\delta Q>0, T_{1}$ is higher than $T_{2}$ according to the second law of thermodynamics and eq. (13) is negative, indicating that there must be entransy dissipation.

The above discussions show that irreversibility of heat transfer processes can also be reflected by entransy dissipation besides entropy generation.
Entransy dissipation can be derived from the energy equation. For a transient heat conduction process in a certain element, the governing equation is

$$
\rho c_{\mathrm{v}} \frac{\partial T}{\partial t}=-\nabla \cdot q+\dot{Q}
$$

where $\rho$ is density, $c_{\mathrm{v}}$ is heat capacity, $t$ is the time, $q$ is heat flux, and $\dot{Q}$ is heat source. Multiplying the equation with $T$, we can get [10]

$$
\rho c_{\mathrm{v}} T \frac{\partial T}{\partial t}=q \nabla T-\nabla \cdot(q T)+\dot{Q} T .
$$

According to the Fourier's Law and the definition of entransy, there is

$$
\begin{aligned}
\lambda(\nabla T)^{2} & =-q \nabla T \\
& =-\rho c_{\mathrm{v}} T \frac{\partial T}{\partial t}-\nabla \cdot(q T)+\dot{Q} T \\
& =[-\nabla \cdot(q T)+\dot{Q} T]-\frac{\partial G}{\partial t}>0
\end{aligned}
$$

where $\lambda$ is thermal conductivity. The two terms in the square bracket are the net entransy from the boundaries and heat sources, the last term (without minus sign) is the entransy increment. Eq. (16) indicates that the entransy in a differential element is not conserved. Part of the entransy from boundaries and heat sources is converted to the entransy of the differential element, and the rest is dissipated. Integrating eq. (16) over time and volume, and applying the Gauss theorem yield

$$
\begin{aligned}
& \int_{t} \int_{V} \lambda(\nabla T)^{2} \mathrm{~d} t \mathrm{~d} V \\
& =\left[-\int_{t} \int_{A}(q T) \mathrm{d} t \mathrm{~d} A+\int_{t} \int_{V} \dot{Q} T \mathrm{~d} t \mathrm{~d} V\right]-\int_{t} \int_{V} \frac{\partial G}{\partial t} \mathrm{~d} t \mathrm{~d} V>0,
\end{aligned}
$$

where $V$ is the volume of interest and $A$ is its surface area. The integrated terms are of the same meaning as those of the differential element. Therefore, entransy dissipation always accompanies heat conduction processes.

For heat convection processes, the governing equation is

$$
\rho c_{\mathrm{v}} \frac{\partial T}{\partial t}+\rho c_{\mathrm{v}} \boldsymbol{u} \cdot \nabla T=-\nabla \cdot q+\dot{Q}+\Phi,
$$

where $\boldsymbol{u}$ is fluid velocity vector, $\Phi$ is viscous dissipation. Similarly to heat conduction, there is

$$
\lambda(\nabla T)^{2}=-\frac{1}{2} \rho c_{\mathrm{v}} \boldsymbol{u} \cdot \nabla\left(T^{2}\right)-\nabla \cdot(q T)+\dot{Q} T+\Phi T-\frac{\partial G}{\partial t}>0,
$$

where the first term (including minus sign) on the right hand side is the entransy with the fluid into the differential element, the second term (including minus) is the entransy from the boundaries, the third term is the entransy flux from 
the heat sources, the forth term is the entransy due to viscous dissipation, and the last term (without minus sign) is entransy increment with time. Obviously, the entransy in a differential element is not conserved, either. Not all the entransy from the boundaries and heat sources is converted to the entransy of the differential element, and the entransy dissipation always exists. Integration of the equation over the whole district leads to the same conclusion.

Entropy generation and entransy dissipation both exist in heat transfer processes and both of them can describe the irreversibility of the heat transfer processes.

Futhermore, the concept of entransy dissipation was found to be more appropriate than the concept of entropy generation $[10,16,20,25,33]$ in optimizing heat transfer processes. For instance, Guo et al. [20] defined the concept of thermal resistance based on the concept of entransy dissipation. When the concept of thermal resistance is used to analyze heat exchangers, researches show that the effectiveness decreases monotonously with the increase in thermal resistance, and there is no paradox similar to the entropy generation paradox [34]. Chen et al. [25] found that the extremum of entransy dissipation rate corresponds to the optimal heat transfer performance of heat exchangers while the minimum entropy generation rate does not.

\subsection{Work-heat conversion process}

The sketch of a work-heat conversion process is shown in Figure 2. Assume that work $\delta W$ is totally converted to heat that is absorbed by the object whose initial temperature is $T$, and finally become its internal energy. The changes of entropy and entransy are analyzed for the finite and infinite heat capacity cases, respectively.

First, let us assume the heat capacity of the object to be finite, and the heat capacity and the mass are $c$ and $m$ respectively. The initial temperature is $T$. According to the first law of thermodynamics, the increment of internal energy is $\delta W$. Based on the relationship of internal energy and temperature $U=c m T$, the temperature becomes

$$
T^{\prime}=(\mathrm{cm} T+\delta W) /(\mathrm{cm}) \text {. }
$$

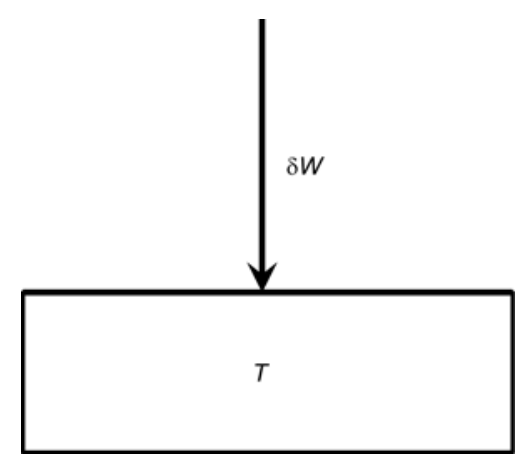

Figure 2 Sketch of a work-heat conversion process
The entropy change in the work-heat conversion process is

$$
\Delta S=\int_{T}^{T^{\prime}} \frac{c m \mathrm{~d} T}{T}=c m \ln \frac{T^{\prime}}{T}=c m \ln \frac{c m T+\delta W}{c m T} .
$$

Eq. (21) is positive for $\delta W>0$. The entropy increases in the work-heat conversion process and there is entropy generation. According to the definition of entransy in eq. (1), the entransy before and after work-heat conversion is

$$
\begin{gathered}
G_{0}=\frac{1}{2} c m T^{2}, \\
G=\frac{1}{2} c m T^{\prime 2}=\frac{1}{2} c m\left(T+\frac{\delta W}{c m}\right)^{2} .
\end{gathered}
$$

The entransy change after work-heat conversion is

$$
\Delta G=G-G_{0}=T \delta W+\frac{\delta W^{2}}{2 c m}>0 .
$$

The entransy does not decrease but increases during the work-heat conversion process. Cheng et al. [35] defined the entransy change due to work input or output as work entransy flow, which is expressed as

$$
\delta G_{\mathrm{W}}=T \delta W .
$$

The increased entransy in the work-heat conversion process is just due to the work entransy.

For the infinite heat capacity case, temperature remains in work-heat conversion process. The entropy change after heat-work conversion is

$$
\Delta S=\int_{0}^{\delta W} \frac{\delta Q}{T}=\frac{\delta W}{T}>0 .
$$

There is entropy generation in the work-heat conversion process. Considering that the heat capacity is infinite, the entransy change is

$$
\Delta G=T \delta W>0 .
$$

We can see that entransy also increases, and the increment is due to the work entransy.

According to the above discussions, there is entropy generation in the work-heat conversion process, which indicates that the physical process is irreversible. However, there is no entransy dissipation. Therefore, the concept of entransy dissipation cannot reflect the irreversibility of the work-heat conversion process.

\subsection{Free expansion process}

Figure 3 is an adiabatic free expansion process of ideal gas. The volume on the right side part of the plate in an adiabatic container is $V_{1}$ and is full of ideal gas whose initial temperature is $T$. The left side part of the plate is vacuum. The whole volume of the container is $V_{2}$. The free expansion 


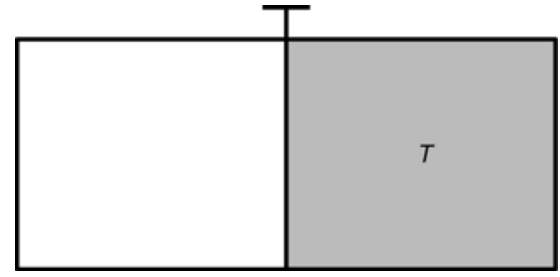

Figure 3 Sketch of a free expansion process

process begins once the plate is taken away.

Entropy is a state quantity and it only depends on the state before and after any process. We can assume a reversible process to calculate the entropy change of the free expansion process. As the temperature remains constant during the process, we take it as a reversible isothermal process whose temperature is $T$, and the ideal gas expands to $V_{2}$ from $V_{1}$. Assume that the mass of ideal gas is $m$, the ideal gas equation is

$$
p V=m R_{\mathrm{g}} T,
$$

where $p$ is the pressure, $V$ is the volume, $m$ is the gas mass, and $R_{\mathrm{g}}$ is the gas constant that depends on the gas type instead of the gas state. The internal energy remains constant during the isothermal process. Therefore, the entropy change in free expansion process is [36]

$$
\Delta S=\int m\left(c \frac{\mathrm{d} T}{T}+R_{\mathrm{g}} \frac{\mathrm{d} V}{V}\right)=m R_{\mathrm{g}} \ln \frac{V_{2}}{V_{1}} .
$$

As $V_{2}>V_{1}$, eq. (29) is positive, and there is entropy generation in the free expansion process. The process is irreversible.

However, in terms of entransy, there is no output work during the expansion process because the left side of the plate is vacuum. According to the first law of thermodynamics, the internal energy of the ideal gas remains constant because the whole system is adiabatic. As the internal energy only includes the average kinetic energy of molecules and the temperature is the measure of the average kinetic energy of molecules, the temperature of the gas after the expansion is the same as that before the expansion. The internal energy and the temperature of the ideal gas before and after the free expansion satisfy

$$
U=U^{\prime}, T=T^{\prime} .
$$

According to the definition of entransy in eq. (1), the entransy before and after the expansion satisfies

$$
G=G^{\prime},
$$

so there is not entransy dissipation during the adiabatic free expansion process of ideal gas.

The adiabatic free expansion process of ideal gas is irreversible but there is no entransy dissipation, which indicates that entransy dissipation cannot reflect the irreversibility of the adiabatic free expansion process of ideal gas.

\subsection{Isothermal diffusion process}

Figure 4 is a diffusion process. Both parts of beside the plate are full of different gases that are not mixed with each other. The volumes of the two parts beside the plate are $V_{1}$ and $V_{2}$ respectively. Assume that the gases in the two parts have the same initial temperature $T$, and the same initial pressure. The gas mass of the two parts is $m_{1}$ and $m_{2}$, respectively. The gas constants are $R_{\mathrm{g} 1}$ and $R_{\mathrm{g} 2}$ respectively. When the plate is taken away, diffusion process happens spontaneously.

Qin [37] pointed out that the diffusion is the superposition of two free expansion processes. The diffusion process shown in Figure 4 is the superposition of the two free expansion process, the one of ideal gas with mass $m_{1}$ and gas constant $R_{\mathrm{g} 1}$ from $V_{1}$ to $V_{1}+V_{2}$ and the one of ideal gas with mass $m_{2}$ and gas constant $R_{\mathrm{g} 2}$ from $V_{2}$ to $V_{1}+V_{2}$. According to eq. (29), the entropy change of the superposition system of the two free expansion processes is

$$
\begin{aligned}
\Delta S & =\Delta S_{1}+\Delta S_{2} \\
& =m_{1} R_{\mathrm{g} 1} \ln \frac{V_{1}+V_{2}}{V_{1}}+m_{2} R_{\mathrm{g} 2} \ln \frac{V_{1}+V_{2}}{V_{2}}>0 .
\end{aligned}
$$

There is an entropy generation in this process, so the process is irreversible.

However, in terms of entransy, the entransy of the whole system remains constant during this free expansion as two gases in the adiabatic system have the same initial temperatures and pressures, and the temperatures of the gases remain constant. There is no entransy dissipation in the diffusion process in Figure 4. Therefore, the concept of entransy dissipation cannot reflect the irreversibility of the isothermal diffusion process. It is necessary to use the concept of mass entransy dissipation that is the extended concept of entransy dissipation to analyze the irreversibility of the diffusion processes [28]. Chen et al. [28] showed that the mass entransy dissipation could measure the irreversibility of mass transfer process, and the relevant theory can optimize convective mass transfer process effectively.

The above analyses of the thermodynamic processes demonstrate that there must be entropy generation in irreversible thermodynamic processes. However, the entransy dissipation does not always exist. Entropy generation can reflect irreversibility of all the thermodynamic processes, while the concept of entransy dissipation cannot. In heat

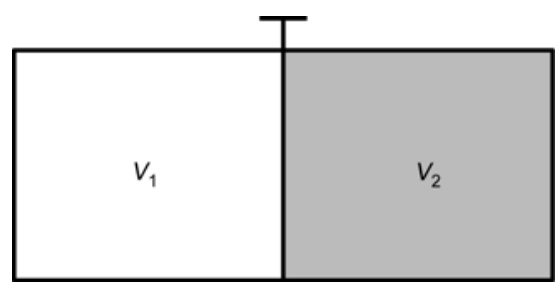

Figure 4 Sketch of isothermal diffusion process. 
transfer processes, entropy generation and entransy dissipation both exist, so entransy dissipation can describe the irreversibility of heat transfer processes.

\section{Analysis of the irreversibility of endoreversible cycles}

The above discussions show that the concept of entransy dissipation can only reflect irreversibility of heat transfer processes. For the thermodynamic cycles including both work output and irreversible heat transfer process, could the irreversibility be described by the concept of entransy dissipation? The irreversibility of a common thermodynamic cycle may be caused by many factors, such as the heat transfer between the working medium and the heat sources, and the friction in heat engines. According to the above analyses, the entransy dissipation could not reflect the irreversibility of the work-heat conversion process, such as friction. However, the irreversibility of the heat transfer processes in thermodynamic cycles can be reflected by entransy dissipation.

Considering that time is an important parameter, Andresen et al. [38-40] developed the finite time thermodynamics, in which the endoreversible cycles are widely used [41]. For the endoreversible cycles, the working medium undergoes quasi-static reversible process, and the irreversibility from the heat transfer, the friction and the vortex are all treated as the thermal resistance loss between the working medium and the heat sources. The entransy dissipation and the irreversibility of the endoreversible cycles are analyzed and discussed below.

The heat transfer law between the working medium and the heat sources in the endoreversible cycles satisfies [42]

$$
Q \propto\left[\Delta\left(T^{n}\right)\right]^{m},
$$

where $Q$ is the heat transfer rate, $n$ and $m$ are constant. Assume that the temperatures of the hot stream and the cold stream are $T_{\mathrm{H}}$ and $T_{\mathrm{L}}$ respectively. The high and the low temperatures of the working medium are $T_{\mathrm{HC}}$ and $T_{\mathrm{LC}}$. According to the heat transfer law, the heat transfer rates between the working medium and the heat sources are

$$
\begin{aligned}
& Q_{\mathrm{H}}=k_{\mathrm{H}}\left(T_{\mathrm{H}}^{n}-T_{\mathrm{HC}}^{n}\right)^{m}, \\
& Q_{\mathrm{L}}=k_{\mathrm{L}}\left(T_{\mathrm{LC}}^{n}-T_{\mathrm{L}}^{n}\right)^{m},
\end{aligned}
$$

where $k_{\mathrm{H}}$ is the heat transfer coefficient between the working medium and the hot stream, $k_{\mathrm{L}}$ is that between the working medium and the cold stream.

As the working medium undergoes reversible cycles, the entropy generation and entransy dissipation of the reversible cycles are both zero, and the entropy generation only exists in the heat transfer processes between the heat sources and the working medium. According to eqs. (34) and (35), the entropy generation rate of this endoreversible cycle is

$$
\dot{S}_{\mathrm{g}}=Q_{\mathrm{H}}\left(\frac{1}{T_{\mathrm{HC}}}-\frac{1}{T_{\mathrm{H}}}\right)+Q_{\mathrm{L}}\left(\frac{1}{T_{\mathrm{L}}}-\frac{1}{T_{\mathrm{LC}}}\right) .
$$

The second law of thermodynamics requires that $T_{\mathrm{H}}>T_{\mathrm{HC}}$ and $T_{\mathrm{LC}}>T_{\mathrm{L}}$. Hence, eq.(36) is positive. Meanwhile, the entransy dissipation rate of this endoreversible cycle is

$$
\dot{G}_{\mathrm{dis}}=Q_{\mathrm{H}}\left(T_{\mathrm{H}}-T_{\mathrm{HC}}\right)+Q_{\mathrm{L}}\left(T_{\mathrm{LC}}-T_{\mathrm{L}}\right) .
$$

Eq.(37) is positive too according to the second law of thermodynamics. Both entropy generation and entransy dissipation exist at the same time in the endoreversible cycles and they both can describe the irreversibility of the endoreversible cycles.

An endoreversible Carnot cycle is discussed as an example below. The endoreversible Carnot cycle was first proposed by Curzon and Ahlborn [43], in which the working medium undergoes the Carnot cycle. Recently, researchers have paid attentions to the characteristics and the optimization of endoreversible Carnot engine which satisfies the generalized heat transfer law [44-46]. In the endoreversible cycle shown in Figure 5, the working fluid takes heat rate $Q_{\mathrm{H}}$ from the hot stream whose temperature is $T_{\mathrm{H}}$, and releases heat rate $Q_{\mathrm{L}}$ to the cold stream whose temperature is $T_{\mathrm{L}}$. The output power of the cycle is $W$. The temperatures of the streams and the working medium satisfy $T_{\mathrm{H}}>T_{\mathrm{HC}}>T_{\mathrm{LC}}>$ $T_{\mathrm{L}}$. According to the first law of thermodynamics, there is

$$
W=Q_{\mathrm{H}}-Q_{\mathrm{L}} .
$$

The relationship between the temperatures and the heat transfer rates is

$$
\frac{Q_{\mathrm{H}}}{T_{\mathrm{HC}}}=\frac{Q_{\mathrm{L}}}{T_{\mathrm{LC}}}
$$

Substituting eqs. (34), (35) into eq. (39), we get the relationship between $T_{\mathrm{HC}}$ and $T_{\mathrm{LC}} . T_{\mathrm{LC}}$ can be determined if $T_{\mathrm{HC}}$ is

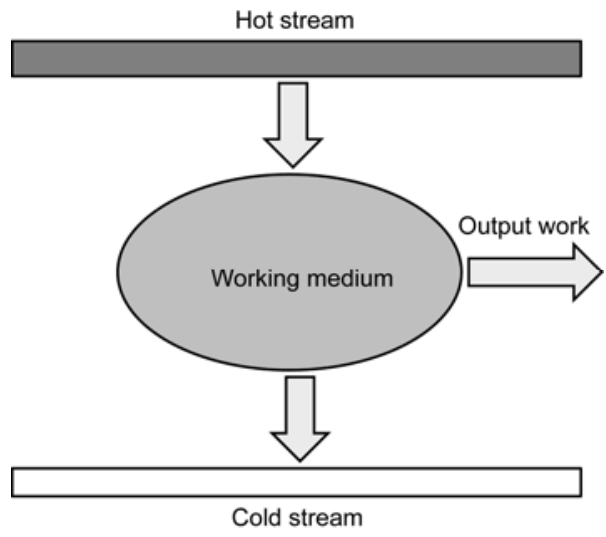

Figure 5 Sketch of an endoreversible Carnot cycle. 
prescribed. Combining (34)-(39) gives the entropy generation rate and the entransy dissipation rate of the endoreversible cycle:

$$
\begin{gathered}
\dot{S}_{\mathrm{g}}=k_{\mathrm{H}}\left(T_{\mathrm{H}}^{n}-T_{\mathrm{HC}}^{n}\right)^{m}\left(\frac{1}{T_{\mathrm{HC}}}-\frac{1}{T_{\mathrm{H}}}\right) \\
+k_{\mathrm{L}}\left(T_{\mathrm{LC}}^{n}-T_{\mathrm{L}}^{n}\right)^{m}\left(\frac{1}{T_{\mathrm{L}}}-\frac{1}{T_{\mathrm{LC}}}\right), \\
\dot{G}_{\mathrm{dis}}=k_{\mathrm{H}}\left(T_{\mathrm{H}}^{n}-T_{\mathrm{HC}}^{n}\right)^{m}\left(T_{\mathrm{H}}-T_{\mathrm{HC}}\right)+k_{\mathrm{L}}\left(T_{\mathrm{LC}}^{n}-T_{\mathrm{L}}^{n}\right)^{m}\left(T_{\mathrm{LC}}-T_{\mathrm{L}}\right) .
\end{gathered}
$$

The heat-work conversion efficiency of this cycle is

$$
\eta=\frac{W}{Q_{\mathrm{H}}} .
$$

Assume that the temperatures of the streams $T_{\mathrm{H}}=400 \mathrm{~K}$, $T_{\mathrm{L}}=300 \mathrm{~K}$, the heat transfer coefficients $k_{\mathrm{H}}=3 \mathrm{~W} \mathrm{~K} \mathrm{~K}^{-m n}, k_{\mathrm{L}}=$ $2.5 \mathrm{~W} \mathrm{~K}^{-m n}$, the heat transfer processes satisfy the generalized heat transfer law in which $m=1.5$ and $n=1.2$. The variations of the efficiency, the entropy generation rate and the entransy dissipation rate with $T_{\mathrm{HC}}$ are shown in Figure 6. The entropy generation rate and entransy dissipation rate decreases and the cycle efficiency increases monotonously with increasing $T_{\mathrm{HC}}$, leading to lower cycle irreversibility. The concepts of entropy generation and entransy dissipation are equivalent when they are used to measure the irreversibility of the endoreversible cycles.

We furthermore change the factors of $m$ and $n$ in the generalized heat transfer law. The variations of the efficiency, the entropy generation rate and the entransy dissipation rate with $T_{\mathrm{HC}}$ are shown in Figure 7 and Figure 8 where the factors are $m=1.5, n=0.8$, and $m=0.7, n=1.2$ respectively. It is shown that the change of $m, n$ does not affect the variation tendencies of the efficiency, the entropy generation rate and the entransy dissipation rate, but only their values. Therefore, the conclusion is appropriate for any heat transfer

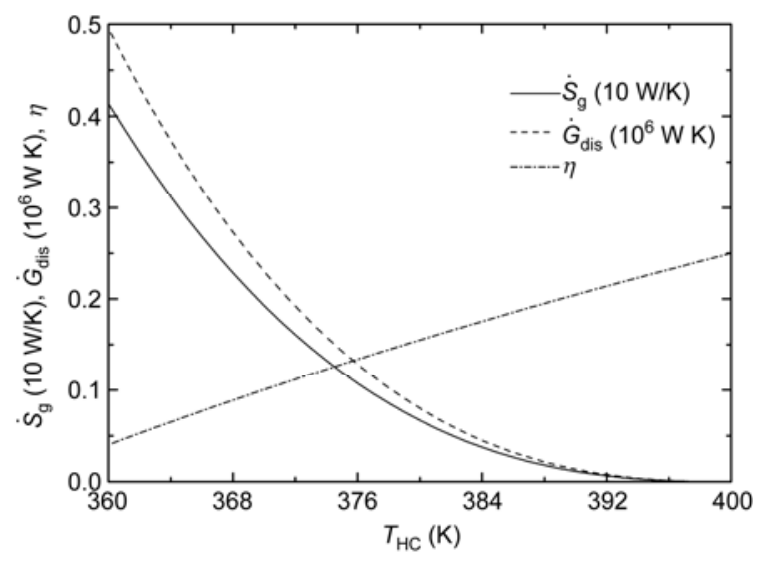

Figure 6 The variations of the efficiency, the entropy generation rate and the entransy dissipation rate with $T_{\mathrm{HC}}$.

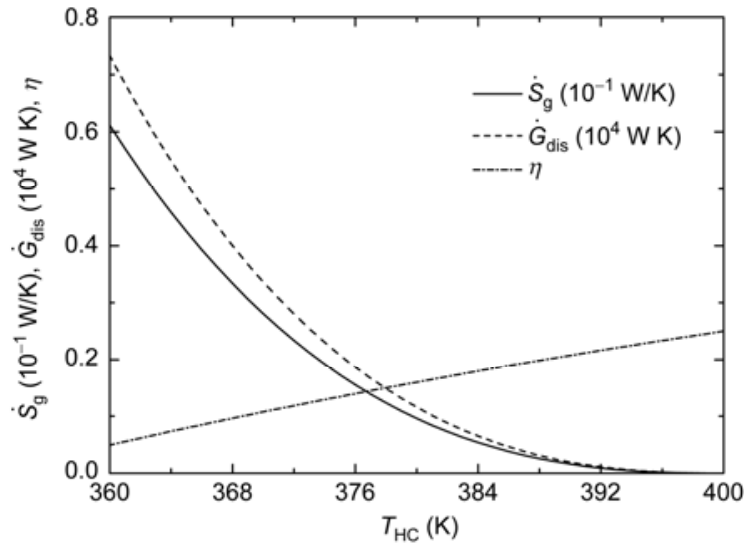

Figure 7 The variations of the efficiency, the entropy generation rate and the entransy dissipation rate with $T_{\mathrm{HC}}$ with different $m, n(m=1.5, n=0.8)$.

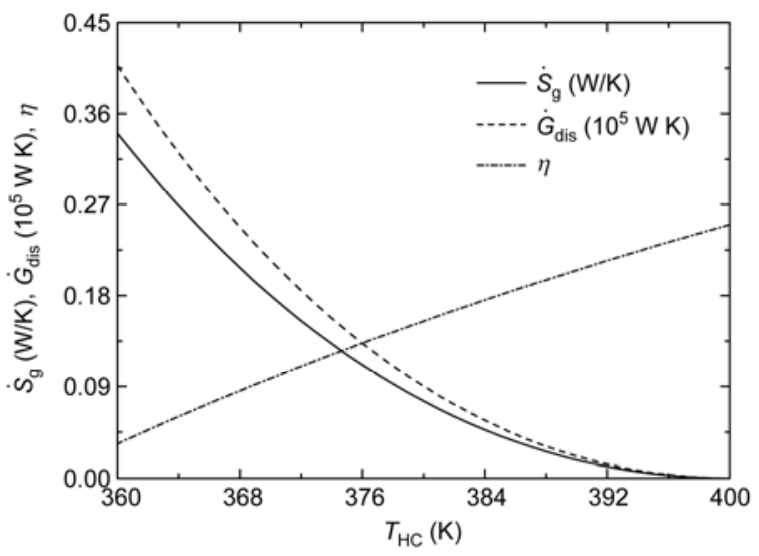

Figure 8 The variations of the efficiency, the entropy generation rate and the entransy dissipation rate with $T_{\mathrm{HC}}$ with different $m, n(m=1, n=1.2)$.

conditions that both the concepts of entropy generation and entransy dissipation can reflect the irreversibility of the endoreversible cycles.

\section{Conclusion}

The irreversibility of heat transfer, work-heat conversion, free expansion, and isothermal diffusion processes is discussed from the viewpoints of entropy and entransy. The results show that entropy generation exists in all the processes, which means these processes are irreversible. However, entransy dissipation does not exist in the work-heat conversion, the free expansion and the isothermal diffusion processes. The analyses of heat transfer, the heat transfer in isolated system, the heat conduction and the heat convection show that entransy is not conserved in heat transfer processes and there must be entransy dissipation. The entransy dissipation can only describe the irreversibility of the heat transfer processes, other than other thermodynamic processes. 
Furthermore, the endoreversible cycles widely used in finite time thermodynamics are analyzed in this paper, which includes the heat transfer process and the work output process. As the irreversibilities are totally attributed to the thermal resistance between the working medium and the heat sources in the endoreversible cycles, the entropy generation and the entransy dissipation both exist in this kind of cycles, and the entransy dissipation can describe their irreversibilities. The variations of the cycle efficiency, the entropy generation rate, and the entransy dissipation rate with the top temperature of the working medium are calculated for an endoreversible Carnot cycle. The results show that the cycle efficiency increases monotonously with the decrease in the entropy generation rate and entransy dissipation rate. The numerical results verify that the concept of entransy dissipation can describe the irreversibilities of the endoreversible cycles.

This work was supported by the National Natural Science Foundation of China (51136001) and the Tsinghua University Initiative Scientific Research Program.

1 Zhao K H, Luo W Y. Thermotics (in Chinese). Beijing: Higher Education Press, 2002

2 Wei W, Sun Q J, Nu R L, et al. The deduction on irreversible equivalent of thermodynamic process. J Xinjiang Normal Univ (Nat Sci Ed), 2010, 29: 55-58

3 Moran M J. Availability Analysis: A Guide to Efficient Energy Use. New Jersey: Prentice-Hall, Inc., 1982

4 Zeng D L, Ao Y, Zhang X M, et al. Engineering Thermodynamics (in Chinese). Beijing: High Eduction Press, 2002

5 Guan H. The history and Current Status of Atomism: The Development of Understanding to Micro-Structure of Materials (in Chinese). Beijing: Peking University Press, 2006

6 Guo Z Y, Zhu H Y, Liang X G. Entransy-A physical quantity describing heat transfer ability. Int J Heat Mass Transfer, 2007, 50: 2545-2556

7 Han G Z, Guo Z Y. Physical mechanism of heat conduction ability dissipation and its analytical expression (in Chinese). Proc CSEE, 2007, 27: 98-102

8 Cheng X T, Liang X G, Xu X H. Microscopic expression of entransy. Acta Phys Sin, 2011, 60: 060512

9 Cheng X T, Liang X G, Guo Z Y. Entransy decrease principle of heat transfer in an isolated system. Chin Sci Bull, 2011, 56: 847-854

10 Cheng X G. Entransy and its applications in heat transfer optimization (in Chinese). Dissertation for Doctoral Degree. Beijing: Tsinghua University, 2004

11 Zhu H Y. The minimum thermal resistance principle based on entransy dissipation (in Chinese). Dissertation for the Doctoral Degree. Beijing: Tsinghua University, 2007

12 Cheng X T, Xu X H, Liang X G. Homogenization of temperature field and temperature gradient field. Sci China Ser E-Tech Sci, 2009, 52: 2937-2942

13 Xie Z H, Chen L G, Sun F R. Constructal optimization for geometry of cavity by taking entransy dissipation minimization as objective. Sci China Ser E-Tech Sci, 2009, 52: 3413-3504

14 Xiao Q H, Chen L G, Sun F R. Constructal entransy dissipation rate minimization for "disc-to-point" heat conduction. Chin Sci Bull, 2011, 56: 102-112

15 Chen Q, Ren J X. Generalized thermal resistance for convective heat transfer and its relation to entransy dissipation. Chin Sci Bull, 2008, 53: $3753-3761$
16 Cheng X T, Liang X G. Entransy flux of thermal radiation and its application to enclosures with opaque surfaces. Int J Heat Mass Transfer, 2011, 54: 269-278

17 Cheng X T, Xu X H, Liang X G. Radiative entransy flux in enclosures with non-isothermal or non-grey, opaque, diffuse surfaces and its application. Sci China-Tech Sci, 2011, 54: 2446-2456

18 Xia S J, Chen L G, Sun F R. Optimization for entransy dissipation minimization in heat exchanger. Chin Sci Bull, 2009, 54: 35723578

19 Guo J F, Xu M T, Cheng L. Principle of equipartition of entransy dissipation for heat exchanger design. Sci China Ser E-Tech Sci, 2010, 53: 1309-1314

20 Guo Z Y, Liu X B, Tao W Q, et al. Effectiveness-thermal resistance method for heat exchanger design and analysis. Int $\mathrm{J}$ Heat Mass Transfer, 2010, 53: 2877-2884

21 Guo J F, Xu M T, Chen L. The entransy dissipation minimization principle under given heat duty and heat transfer area conditions. Chin Sci Bull, 2011, 56: 2071-2076

22 Li X F, Guo J F, Xu M T, et al. Entransy dissipation minimization for optimization of heat exchanger design. Chin Sci Bull, 2011, 56: 2174-2178

23 Qian X D, Li Z X. Analysis of entransy dissipation in heat exchangers. Int J Therm Sci, 2011, 50: 608-614

24 Cheng X T, Xu X H, Liang X G. Application of entransy to optimization design for parallel thermal network of thermal control system in spacecraft. Sci China-Tech Sci, 2011, 54: 964-971

25 Chen Q, Wu J, Wang M R, et al. A comparison of optimization theories for energy conservation in heat exchanger groups. Chin Sci Bull, 2011, 56: 449-454

$26 \mathrm{Xu}$ M T. The thermodynamic basis of entransy and entransy dissipation. Energy, 2011, 36: 4272-4277

27 Bernardes M. Developments in Heat Transfer. Rijeka: In Tech-Open Access Publisher, 2011. 247-272

28 Chen Q, Ren J X, Guo Z Y. The extremum principle of mass entransy dissipation and its application to decontamination ventilation designs in space station cabins. Chin Sci Bull, 2009, 54: 2862-2870

29 Xia S J, Chen L G, Sun F R. Entransy dissipation minimization for a class of one-way isothermal mass transfer processes. Sci China-Tech Sci, 2011, 54: 352-361

30 Cheng X T, Dong Y, Liang X G. Potential entransy and potential entransy decrease principle. Acta Phys Sin, 2011, 60: 114402

31 Cheng X T, Xu X H, Liang X G. Principles of potential entransy in generalized flow. Acta Phys Sin, 2011, 60: 118103

32 Zeng D L. The Development of Non-Equilibrium Thermodynamics (in Chinese). Beijing: Science Press, 2009

33 Wu J, Cheng X G, Meng J A, et al. Potential capacity dissipation extremum and entropy generation minimization in laminar convective heat transfer (in Chinese). J Eng Thermophys, 2006, 27: 100-102

34 Bejan A. Advanced Engineering Thermodynamics. 2nd ed. New York: John Wiley \& Sons, 1997

35 Cheng X T, Liang X G. Entransy loss in thermodynamic processes and its application. Energy, 2012, 44: 964-972

36 Shen W D, Tong J G. Engineering Thermodynamics (in Chinese). 4th ed. Beijing: High Eduction Press, 2007

37 Qin Y H. Thermotics (in Chinese). Beijing: Higher Education Press, 2004

38 Andresen B, Berry R S, Nitzan A, et al. Thermodynamics in finite time I: The step Carnot cycle. Phys Rev A, 1977, 15: 2086-2093

39 Salamon P, Andresen B, Berry R S. Thermodynamics in finite time II: Potentials for finite time processes. Phys Rev A, 1977, 15: 20942101

40 Berry R S, Salamon P, Heal G. On a relation between economic and thermodynamic optima. Resources Energ, 1978, 1: 125-137

41 Chen L G, Sun F R. Finite time thermodynamic theory and applications: State of the arts (in Chinese). Prog Phys, 1998, 18: 395-422

42 Xia S J, Chen L G, Sun F R. Optimization for minimizing entropy generation during heat transfer processes with heat transfer law $q \propto$ $\left(\Delta\left(T^{n}\right)\right)^{m}$ (in Chinese). J Therm Sci Tech, 2008, 7: 226-230 
43 Curzon F L, Ahlborn B. Efficiency of a Carnot engine at maximum power output. Am J Phys, 1975, 43: 22-24

44 Li J, Chen L G, Sun F R. Optimal configuration for a finite high-temperature source heat engine cycle with the complex heat transfer law. Sci China Ser G-Phys Mech Astron, 2009, 52: 587-592
45 Li J, Chen L G, Sun F R, et al. Power vs. efficiency characteristic of an endoreversible Carnot heat engine with heat transfer law $q \propto$ $\left(\Delta\left(T^{n}\right)\right)^{m}$. Int J Ambient Energ, 2008, 29: 149-152

46 Chen L G, Li J, Sun F R. Generalized irreversible heat-engine experiencing a complex heat-transfer law. Appl Energ, 2008, 85: 52-60

Open Access This article is distributed under the terms of the Creative Commons Attribution License which permits any use, distribution, and reproduction in any medium, provided the original author(s) and source are credited. 